\title{
Negative impact of proteinuria on circulating myeloid dendritic cells
}

\author{
Masato Ikeda ${ }^{1}\left(\mathbb{D} \cdot\right.$ Risa Terashima $^{2} \cdot$ Taku Yamada $^{1} \cdot$ Masahiro Suyama $^{1} \cdot$ Shinya Yokote $^{1} \cdot$ Masatsugu Nakao $^{2}$. \\ Izumi Yamamoto $^{2} \cdot$ Keita Hirano $^{2} \cdot$ Hideo Okonogi $^{2} \cdot$ Hiroyasu Yamamoto $^{2} \cdot$ Takashi Yokoo $^{2}$
}

Received: 27 September 2018 / Accepted: 1 March 2019 / Published online: 16 March 2019

(c) The Author(s) 2019

\begin{abstract}
Background A decrease in absolute numbers (abs.) of circulating dendritic cells (DCs) and recruitment into target organs has been reported, but whether the level of proteinuria associates with circulating DC abs. has not been clarified.

Methods We conducted a cross-sectional study of 210 patients with kidney disease aged 21-96 years who were admitted to our hospital for kidney biopsy in 2007-2010. For accuracy, the level of proteinuria was thoroughly measured by 24-h urine collection from patients in their admitted condition. The abs. of total DCs (tDCs), myeloid DCs (mDCs) and plasmacytoid DCs (pDCs) was measured by three-color fluorescence-activated cell sorting (FACS). Patients were divided into four groups based upon the quartile of each DC abs. and one-way ANOVA, and multivariable-adjusted regression analyses were performed.

Results Quantile analysis showed that the level of daily proteinuria decreased with increasing blood $\mathrm{mDC}$ abs., with mean proteinuria levels (g/day) of $2.45,1.68,1.68,1.10$ for those in $\mathrm{mDC}$ abs. quartiles $\leq 445,<686,<907, \geq 907$ cells $/ 10^{2} \mu \mathrm{L}$ $(p=0.0277)$, respectively. Multivariate-adjusted regression analysis revealed that the $\mathrm{mDC}$ abs. was negatively associated with proteinuria (95\% CI -57.0 to -8.5 ) and positively associated with male gender (95\% CI 66.2-250.5). Independent associations were also shown between pDCs abs. and estimated glomerular filtration rate (eGFR) (95\% CI 0.14-2.67) and C-reactive protein (95\% CI - 49.4 to - 9.9) and between tDCs abs. and male gender (95\% CI 54.5-253.6) and C-reactive protein $(95 \% \mathrm{CI}-80.5$ to -13.4$)$.
\end{abstract}

Conclusion We first reported that circulating $\mathrm{mDC}$ abs. has a negative association with the level of proteinuria.

Keywords Dendritic cells · Proteinuria · eGFR · Gender · C-reactive protein

\begin{tabular}{|c|c|c|c|c|}
\hline \multicolumn{3}{|c|}{ Abbreviations } & CKD & Chronic kidney disease \\
\hline \multicolumn{2}{|c|}{$\mathrm{DC}$} & Dendritic cell & RRT & Renal replacement therapy \\
\hline \multicolumn{2}{|c|}{$\mathrm{tDC}$} & Total DC & eGFR & Estimated glomerular filtration rate \\
\hline \multicolumn{2}{|c|}{$\mathrm{mDC}$} & Myeloid DC & BMI & Body mass index \\
\hline \multicolumn{2}{|c|}{$\mathrm{pDC}$} & Plasmacytoid DC & $\mathrm{Cr}$ & Creatinine \\
\hline \multicolumn{2}{|c|}{ FACS } & Fluorescence-activated cell sorting & HDL & High-density lipoprotein \\
\hline \multicolumn{2}{|c|}{ abs. } & Absolute number & LDL & Low-density lipoprotein \\
\hline \multicolumn{2}{|l|}{$\mathrm{CI}$} & Confident interval & NK cells & Natural killer cells \\
\hline \multicolumn{2}{|c|}{ DCs } & Dendritic cells & BUN & Blood urea nitrogen \\
\hline \multirow{2}{*}{\multicolumn{2}{|c|}{ CNS }} & Central nervous system & HLA & Human leukocyte antigen \\
\hline & & & $\mathrm{CD}$ & Cluster of differentiation \\
\hline \multirow[t]{3}{*}{$\triangle$} & \multirow{3}{*}{\multicolumn{2}{|c|}{$\begin{array}{l}\text { Masato Ikeda } \\
\text { aoto-jinnai@jikei.ac.jp }\end{array}$}} & IgG & Immunoglobulin $\mathrm{G}$ \\
\hline & & & $\operatorname{IgA}$ & Immunoglobulin A \\
\hline & & & $\operatorname{IgAN}$ & IgA nephropathy \\
\hline \multirow[t]{3}{*}{1} & \multirow{3}{*}{\multicolumn{2}{|c|}{$\begin{array}{l}\text { Division of Nephrology and Hypertension, Department } \\
\text { of Internal Medicine, Katsushika Medical Center, Jikei } \\
\text { University School of Medicine, 6-41-2 Katsushika-ku, } \\
\text { Tokyo 125-8506, Japan }\end{array}$}} & $\mathrm{MN}$ & Membranous nephropathy \\
\hline & & & Minor & Minor glomerular abnormalities \\
\hline & & & CreGN & Crescentic glomerulonephritis \\
\hline \multirow[t]{2}{*}{2} & \multirow{2}{*}{\multicolumn{2}{|c|}{$\begin{array}{l}\text { Division of Nephrology and Hypertension, Department } \\
\text { of Internal Medicine, Jikei University School of Medicine, }\end{array}$}} & FSGS & Focal segmental glomerulosclerosis \\
\hline & & & MCD & Minimal change disease \\
\hline
\end{tabular}




$\begin{array}{ll}\text { ATIN } & \text { Acute tubulointerstitial nephritis } \\ \text { MIP } & \text { Macrophage inflammatory protein } \\ \text { CCL } & \text { CC chemokine ligand } \\ \text { CCR } & \text { CC chemokine receptor }\end{array}$

\section{Introduction}

DCs are the major antigen-presenting cells and are distributed throughout the body including kidney [1]. The subsets of circulating DCs include precursor $\mathrm{mDCs}$, or conventional DCs that initiate T-cell immunity and antibody production, and pDCs, which have an important role in antiviral immunity and immune tolerance.

Several diseases influence circulating DCs, and decreases in circulating DCs and recruitment into target organs have been reported in autoimmune diseases [2-4], infections [5], coronary artery disease [6], central nervous system (CNS) disturbances $[7,8]$ and atherosclerosis, where DCs have been identified in atherosclerotic plaques [9].

In kidney mDCs and pDCs, both subsets usually locate in the tubulointerstitium often with a high frequency [10] and increase in number as the severity of kidney disease increases [11].

A reduction in the number of circulating pDCs has been reported in patients with chronic kidney disease (CKD) compared with that in healthy controls. Several reports have shown that the reduction in $\mathrm{pDC}$ numbers was more striking than that in mDCs [12-14]. However, the reduction in the number of circulating mDCs is controversial. Lim et al. [13] reported that circulating mDCs abs. in dialysis patients was comparable to that in healthy controls. Hesselink et al. [12] also reported that the estimated glomerular filtration rate (eGFR) was correlated with pDC numbers, but not mDC numbers, and these levels were comparable to those of volunteer patients with CKD not receiving dialysis.

Though the level of proteinuria is a sensitive marker for kidney disease, the association between the circulating DCs abs. and the level of proteinuria has not been clarified to date. If the circulating DC abs. decreases with the level of proteinuria, the measurement of blood DC abs. may be a biomarker for renal disease activity.

Considering all the evidence, we evaluated the hypothesis that the circulating DC abs. could be altered by the level of proteinuria, and we found that $\mathrm{mDCs}$ abs. was significantly associated with the level of proteinuria negatively, independent of eGFR. We also found that the number of pDCs, but not the number of mDCs, was associated with eGFR by multivariate regression analysis adjusted for proteinuria, C-reactive protein, and gender.

\section{Methods}

\section{Study design}

This study was a cross-sectional, observational, singlecenter study and comprised 243 Japanese patients who received kidney biopsy between December 1, 2007, and June 31, 2010. Clinical information and hematological data were collected immediately before the kidney biopsy. This study was performed in accordance with the Declaration of Helsinki. The Ethics Committee for Clinical Research of Jikei University School of Medicine approved this study [permission no. 29-032 (8648)].

To explore the association between the abs. of each subset of DCs and clinical data, the following inclusion criteria were selected: (1) adult patients with biopsy-proven kidney disease and (2) patient records with complete data for the following factors: age, gender, body mass index (BMI), glucocorticoid use, level of proteinuria in collected daily urine, and fasting blood samples. As a result, patients were excluded from the analysis due to insufficient laboratory data (33 patients). Therefore, of the 243 patients evaluated, 210 Japanese patients satisfied the inclusion criteria and were included in the analysis (Fig. 1).

eGFR was calculated using Japanese equation [15]: eGFR $\left(\mathrm{mL} / \mathrm{min} / 1.73 \mathrm{~m}^{2}\right)=194 \times \mathrm{Cr}^{-1.094} \times \mathrm{age}^{-0.287}(\times 0.739$ for women).

The primary variable was the abs. of mDCs, pDCs, and total DCs. Predictor variables were the levels of daily proteinuria, eGFR, C-reactive protein, and the other clinical parameters.

\section{3 patients just prior to kidney biopsy}

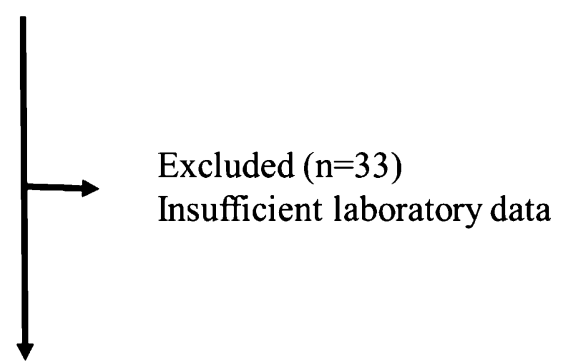

210 patients included in the analysis

Fig. 1 Flow chart of recruitment of study participants. Patients were excluded from the analysis due to insufficient laboratory data (33 patients). Of the 243 patients evaluated, 210 Japanese patients satisfied the inclusion criteria and were included in the analysis 


\section{Flow cytometric identification of DCs}

Peripheral blood samples were collected in the morning during admission for kidney biopsy, and flow cytometric analysis and peripheral blood DC quantification were performed within $3 \mathrm{~h}$ after blood sampling. DCs were identified in heparinized whole-blood samples (Greiner Bio-One, Frickenhausen, Germany) using a combination of fluorochromeconjugated monoclonal antibodies to different membrane markers. Reagents were obtained from BD Biosciences (Erembodegem, Belgium): $20 \mu \mathrm{L}$ lineage cocktail 1-fluorescein isothiocyanate (Lin1-FITC), containing a mixture of anti-cluster of differentiation (CD) 3, CD14, CD16, CD19, CD20, and CD56 antibodies; $5 \mu \mathrm{L}$ anti-CD123-PE; $5 \mu \mathrm{L}$ anti-CD11c-APC; and $10 \mu \mathrm{L}$ anti-human leukocyte antigen (HLA)-DR were incubated with $100 \mu \mathrm{L}$ whole blood for $15 \mathrm{~min}$ at room temperature in the dark. Cells were fixed and lysed for $20 \mathrm{~min}$ at room temperature using FACS lysing solution (BD Bioscience). Cells were centrifuged at $200 \times g$ for $10 \mathrm{~min}$ and resuspended in $0.5 \mathrm{~mL}$ phosphate-buffered saline containing $0.1 \% \mathrm{NaN}_{3}$.

Data acquisition was performed on a three-color FACSCalibur (BD Bioscience) using a well-defined gating strategy in Cell Quest Pro software (Beckton Dickinson, Heidelberg, Germany). At least $5 \times 10^{5}$ cells were measured to obtain a minimum of 1000 HLA-DR-positive DCs. Representative dot plots of the gating strategy for identifying mDCs and pDCs by three-color FACS are shown in Fig. 2. Whole peripheral leukocytes were selected from live cells based on SSC-height and FSC-height characteristics (Fig. 2a, gate R1). Blood DCs were then selected within R1-positive cells as negative for lineage markers (lin-1) (CD3, CD14, CD16, CD19, CD20 and CD56) and positive for HLA-DR (Fig. 2b, gate R2). Next, according to the expression of CD123 and CD11c, blood DCs were identified as mDCs (Fig. 2c, HLA$\mathrm{DR}+/ \mathrm{CD} 11 \mathrm{c}+/ \mathrm{CD} 123 \mathrm{dim}^{+}$, Gate R3) or pDCs (Fig. 2c, HLA-DR+/CD11c-/CD123 high ${ }^{+}$, Gate R4) [8, 16, 17]. Dot plots for the negative control of either CD 123 (Fig. 2d) or CD11c (Fig. 2e) are also shown.

\section{Statistics}

Statistical analyses were performed using JMP 9.0 (SAS Institute Inc., Cary, NC, USA). Data are expressed as the mean \pm standard deviation or numbers (percentage) of patients. Comparisons across the various groups were performed using the Pearson Chi square test for categorical data, Dunnett test for continuous data and ANOVA for quartile analysis. All tests were two-tailed, and a $p$ value of $<0.05$ was considered significant. Factors associated with DC abs. on univariate analysis were subsequently included in a multivariate model. Multivariate regression analyses were performed to identify the covariates associated with each DC abs. $T$ values and 95\% confidence intervals (CIs) were determined using univariate and multivariate logistic regression models for the factors that were significantly associated with each DC abs.

\section{Results}

\section{Histopathological diagnoses and patient characteristics}

Histopathological diagnoses of these patients including $\operatorname{Ig} \mathrm{A}$ nephropathy ( $n=67,31.9 \%)$, benign/malignant nephrosclerosis $(n=31,14.8 \%)$, diabetic nephropathy $(n=26,12.4 \%)$, membranous nephropathy $(n=15,7.1 \%)$, minor glomerular abnormalities $(n=14,6.7 \%)$, crescentic glomerulonephritis $(n=11,5.2 \%)$, focal segmental glomerulosclerosis $(n=11$, $5.2 \%)$, minimal change disease (MCD) $(n=7,3.3 \%)$, lupus nephritis $(n=5,2.4 \%)$, IgA vasculitis $(n=4,1.9 \%)$, obesityrelated nephropathy $(n=4,1.9 \%)$ and others $(n=15,7.1 \%)$ (Table 1). The other patient characteristics and clinical data are shown in Table 2.

\section{DC abs. and quantile analysis}

The mean abs. of tDCs, mDCs, and pDCs were $1421.4 \pm 366.0(326-2766)$ cells $/ 10^{2} \mu \mathrm{L}, 702.1 \pm 336.9$ (61-1952) cells $/ 10^{2} \mu \mathrm{L}$, and $415.5 \pm 219.7(0-1192)$ cells $/ 10^{2} \mu \mathrm{L}$, respectively. The relative numbers of tDCs, mDCs, and pDCs (\% of leukocytes) were $0.24 \pm 0.09$ $(0.04-0.66) \%, 0.12 \pm 0.07(0.01-0.51) \%$, and $0.07 \pm 0.04$ $(0-0.28) \%$.

Quantile analysis showed that the level of daily proteinuria increased with decreasing blood $\mathrm{mDC}$ abs., with mean proteinuria levels (g/day) of $2.45,1.68,1.68,1.10$ for the mDC abs. quartiles of $\leq 445,<686,<907$, and $\geq 907$ cells $/ 10^{2} \mu \mathrm{L}(p=0.0277)$, respectively (Table 3a). Compared to patients with the highest $\mathrm{mDC}$ abs., patients with the lowest $\mathrm{mDC}$ abs. were more often female. The lowest $\mathrm{mDC}$ abs. was also associated with lower serum albumin, higher C-reactive protein, a higher level of daily proteinuria and lower hemoglobin (Table 3a).

Compared to patients with the highest pDC abs., patients with the lowest pDC abs. were older and had lower eGFR, higher BUN, higher serum $\mathrm{Cr}$, higher C-reactive protein and lower hemoglobin (Table 3b).

Compared to patients with the highest tDC abs., patients with the lowest tDC abs. were older and more often female and had lower eGFR, higher blood urea nitrogen (BUN), higher C-reactive protein and lower hemoglobin (Table 3c). 
Fig. 2 Flow cytometric identification of DCs. Representative dot plots showing the gating strategy to identify mDCs and pDCs by flow cytometry. Whole peripheral leukocytes were selected from live cells based on SSC-height and FSC-height characteristics (a, gate R1). Blood DCs were then selected within R1-positive cells as negative for lineage markers (lin-1) (CD3, CD14, CD16, CD19, CD20, and CD56) and positive for HLA-DR. (b, gate R2). Next, according to the expression of CD123 and CD11c, blood DCs were identified as mDCs (c, HLA-DR+/ CD11c+/CD123 dim ${ }^{+}$, Gate R3) or pDCs (c, HLA-DR+/ CD11c-/CD123 high ${ }^{+}$, Gate R4). Dot plots for the negative control (NC) of either CD 123 (d, NC PE) or CD11c (e, NC APC) are also shown
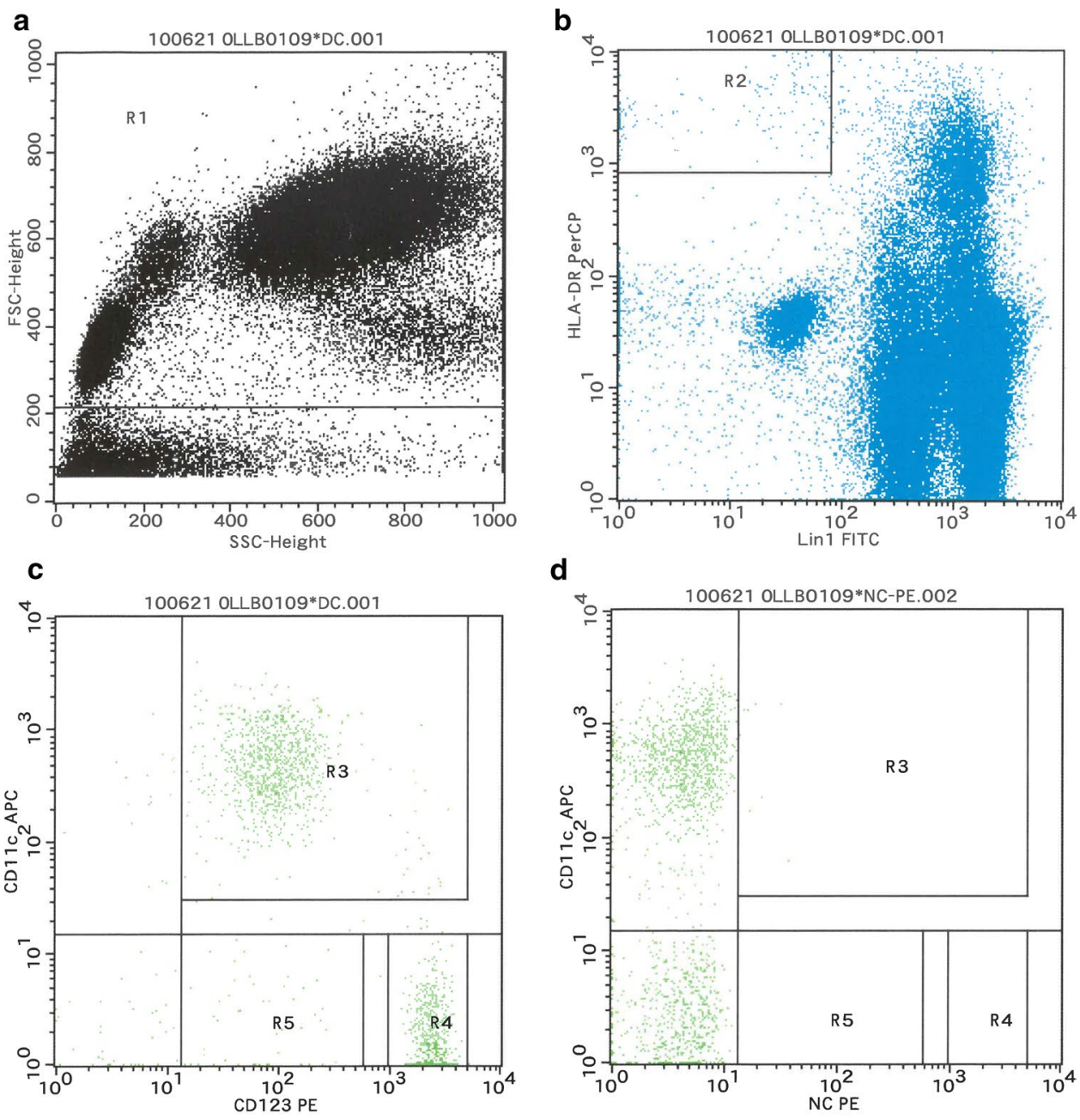

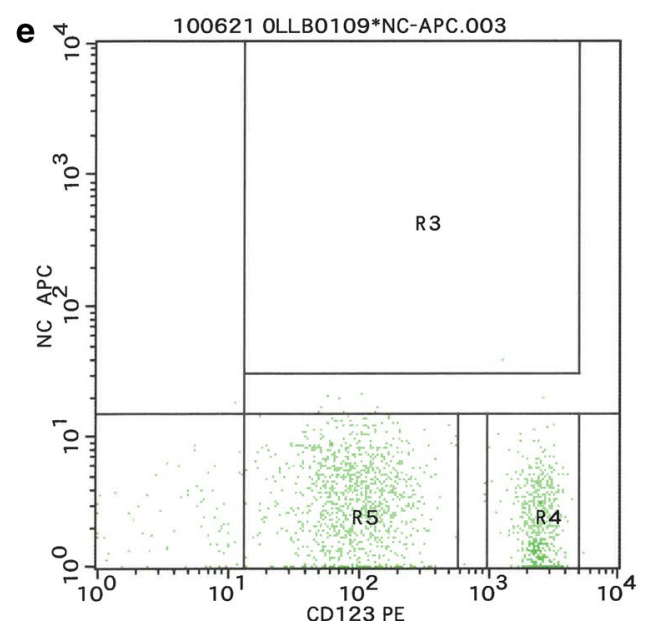

\section{Associations between clinical factors and DC abs.}

As shown in Table 4, univariate and multivariate-adjusted regression analyses were used to assess the independent associations between DC subsets (tDCs, $\mathrm{mDCs}$, and $\mathrm{pDCs}$ ) and explanatory variables.
The primary variables were tDCs abs., mDCs abs. and pDCs abs., and the explanatory variables were determined according to their univariate relationship or importance within the explanatory variables as follows: age, gender, albumin, eGFR, level of daily proteinuria, C-reactive protein and hemoglobin. 
Table 1 Histopathological diagnoses of participants

\begin{tabular}{ll}
\hline & $\begin{array}{l}\text { Number } \\
\text { of patients } \\
(\%)\end{array}$ \\
\hline IgAN & $67(31.9)$ \\
Benign/malignant nephrosclerosis & $31(14.8)$ \\
Diabetic nephropathy & $26(12.4)$ \\
MN & $15(7.1)$ \\
Minor & $14(6.7)$ \\
FSGS & $11(5.2)$ \\
CreGN & $11(5.2)$ \\
MCD & $7(3.3)$ \\
Lupus nephritis & $5(2.4)$ \\
IgA vasculitis & $4(1.9)$ \\
Obesity-related nephropathy & $4(1.9)$ \\
Others & $15(7.1)$ \\
Total & $210(100)$ \\
\hline
\end{tabular}

Number and percentage of histopathological diagnoses including immunoglobulin A nephropathy $(\operatorname{IgAN})(n=67,31.9 \%)$, benign/ malignant nephrosclerosis $(n=31,14.8 \%)$, diabetic nephropathy $(n=26,12.4 \%)$, membranous nephropathy (MN) $(n=15,7.1 \%)$, minor glomerular abnormalities (minor) $(n=14,6.7 \%)$, crescentic glomerulonephritis (CreGN) $(n=11,5.2 \%)$, focal segmental glomerulosclerosis (FSGS) $(n=11,5.2 \%)$, minimal change disease (MCD) $(n=7,3.3 \%)$, lupus nephritis $(n=5,2.4 \%), \operatorname{IgA}$ vasculitis $(n=4$, $1.9 \%)$, obesity-related nephropathy $(n=4,1.9 \%)$ and others $(n=15$, $7.1 \%)$

Multivariate-adjusted regression analysis for $\mathrm{mDCs}$ abs. revealed an independent negative association with the level of proteinuria $(t=-2.66,95 \%$ CI -57.0 to -8.5 , $p=0.0084)$ and a positive association with male gender $(t=3.39,95 \%$ CI 66.2-250.5, $p=0.0008)$ after adjusting for age, serum albumin, eGFR, C-reactive protein, and hemoglobin. Although serum albumin, C-reactive protein and hemoglobin were factors associated with mDCs abs. in univariate logistic regression analyses, these factors were not independent risk factors in multivariate-adjusted regression analysis.

Multivariate-adjusted regression analysis for pDCs abs. revealed an independent positive association with eGFR $(t=2.19,95 \%$ CI $0.14-2.67, p=0.0298)$ and a negative association with C-reactive protein $(t=-2.96,95 \%$ CI -49.4 to $-9.9, p=0.0034$ ) after adjusting for age, male gender, serum albumin, level of proteinuria, and hemoglobin. Although age and hemoglobin were factors associated with pDCs abs. in univariate analyses, these factors were not independent risk factors in multivariate-adjusted logistic regression analysis.

Again, multivariate-adjusted regression analysis (leastsquares method) for tDCs abs. revealed an independent positive association with male gender $(t=3.05,95 \% \mathrm{CI}$ $54.5-253.6, p=0.0026)$ and a negative association with
Table 2 Patients characteristics and clinical data

\begin{tabular}{|c|c|}
\hline & Overall $(n=210)$ \\
\hline Age (years) & $57.6 \pm 16.2(21-96)$ \\
\hline Male, $n(\%)$ & $121(57.6)$ \\
\hline Diabetes mellitus nephropathy, $n(\%)$ & $26(12.4)$ \\
\hline BMI $\left(\mathrm{kg} / \mathrm{m}^{2}\right)$ & $23.5 \pm 3.8(14.7-35.2)$ \\
\hline Glucocorticoids user, $n(\%)$ & $24(11.4)$ \\
\hline Serum albumin $(\mathrm{g} / \mathrm{dL})$ & $3.6 \pm 0.7(1.5-4.7)$ \\
\hline Serum urea nitrogen $(\mathrm{mg} / \mathrm{dL})$ & $24.9 \pm 17.4(6-109)$ \\
\hline Serum creatinine $(\mathrm{mg} / \mathrm{dL})$ & $1.72 \pm 1.39(0.48-8.9)$ \\
\hline eGFR (mL/min/1.73 m²) & $47.1 \pm 28.0(4.5-133.2)$ \\
\hline Serum sodium $(\mathrm{mEq} / \mathrm{L})$ & $141.5 \pm 2.5(133-148)$ \\
\hline Serum potassium $(\mathrm{mEq} / \mathrm{L})$ & $4.3 \pm 0.5(2.5-5.9)$ \\
\hline Serum chloride (mEq/L) & $106.0 \pm 3.8(90-117)$ \\
\hline Serum-corrected calcium (mg/L) & $9.3 \pm 0.6(7-11)$ \\
\hline Serum phosphorus (mg/dL) & $3.5 \pm 0.9(1.6-10.7)$ \\
\hline Serum triglyceride (mg/dL) & $163.0 \pm 92.3(33-710)$ \\
\hline Serum HDL (mg/dL) & $51.9 \pm 16.0(19-110)$ \\
\hline Serum LDL (mg/dL) & $121.8 \pm 40.8(34-299)$ \\
\hline Serum C-reactive protein $(\mathrm{mg} / \mathrm{dL})$ & $0.5 \pm 1.5(0.1-15.7)$ \\
\hline Proteinuria (g/day) & $1.73 \pm 2.32(0.05-13.65)$ \\
\hline Serum IgG (mg/dL) & $1156.9 \pm 389.8(322-3223)$ \\
\hline Serum $\operatorname{IgA}(\mathrm{mg} / \mathrm{dL})$ & $295.1 \pm 119.8(96-1015)$ \\
\hline Leucocyte $\left(10^{3} / \mathrm{mL}^{3}\right)$ & $6526 \pm 2199(1900-22,800)$ \\
\hline Hemoglobin (g/dL) & $12.2 \pm 2.3(7-17.2)$ \\
\hline Platelet $\left(10^{4} / \mu \mathrm{L}\right)$ & $232.5 \pm 73.5(19.4-735)$ \\
\hline
\end{tabular}

Median (min-max) and mean \pm standard deviation are shown

$B M I$ body mass index, $e G F R$ estimated glomerular filtration rate, $H D L$ high-density lipoprotein, $L D L$ low-density lipoprotein

C-reactive protein $(t=-2.76,95 \% \mathrm{CI}-80.5$ to -13.4 , $p=0.0063$ ) after adjusting for age, serum albumin, eGFR, level of proteinuria, C-reactive protein, and hemoglobin.

Although younger age, higher level of eGFR, hemoglobin and lower level of phosphate were factors associated with the tDCs abs. in univariable logistic regression analyses, these factors were not independent risk factors in multivariate-adjusted regression analysis.

Diabetic nephropathy, BMI, glucocorticoid use, sodium, chloride, corrected calcium, phosphate, triglyceride, HDL, LDL, IgG, IgA, leukocytes and platelets were not associated with tDCs, mDCs or pDCs.

\section{Discussion}

The present study demonstrated four factors (proteinuria, eGFR, C-reactive protein, and gender) independently associated with circulating mDC abs., pDC abs. or tDC abs. by multivariate regression analysis as follows. First, 
Table 3 Quartile analysis by each abs. of mDCs (Table 3a), pDCs (Table 3b) and tDCs (Table 3c)

\begin{tabular}{|c|c|c|c|c|c|}
\hline \multirow[t]{2}{*}{ (a) } & \multicolumn{5}{|c|}{ mDC quartiles (cells $/ 10^{2} \mu \mathrm{L}$ ) } \\
\hline & $\leq 445(n=53)$ & $445-686(n=52)$ & $686-907(n=53)$ & $\geq 907(n=52)$ & $p$ for trend \\
\hline Age (years) & $58.7 \pm 15.6$ & $56.4 \pm 18.0$ & $60.7 \pm 14.8$ & $54.5 \pm 16.2$ & 0.2196 \\
\hline Male, $n(\%)$ & $23,43.4 \%$ & $28,53.9 \%$ & $29,54.7 \%$ & $41,78.9 \%$ & 0.0005 \\
\hline Diabetes mellitus nephropathy, $n(\%)$ & $9,17.0 \%$ & $5,9.6 \%$ & $6,23.1 \%$ & $6,11.5 \%$ & 0.4674 \\
\hline $\operatorname{BMI}\left(\mathrm{kg} / \mathrm{m}^{2}\right)$ & $23.1 \pm 4.1$ & $23.0 \pm 3.8$ & $24.1 \pm 3.9$ & $23.8 \pm 3.3$ & 0.3598 \\
\hline Glucocorticoids user, $n(\%)$ & $6,11.3 \%$ & $8,15.4 \%$ & $7,13.2 \%$ & $3,5.8 \%$ & 0.3432 \\
\hline Serum albumin $(g / d L)$ & $3.3 \pm 0.8$ & $3.6 \pm 0.8$ & $3.6 \pm 0.7$ & $3.7 \pm 0.6$ & 0.0162 \\
\hline Serum urea nitrogen $(\mathrm{mg} / \mathrm{dL})$ & $29.5 \pm 21.7$ & $23.0 \pm 16.1$ & $24.8 \pm 15.6$ & $22.0 \pm 14.8$ & 0.1231 \\
\hline Serum creatinine $(\mathrm{mg} / \mathrm{dL})$ & $2.0 \pm 1.8$ & $1.7 \pm 1.3$ & $1.8 \pm 1.6$ & $1.5 \pm 0.7$ & 0.3045 \\
\hline $\mathrm{eGFR}\left(\mathrm{mL} / \mathrm{min} / 1.73 \mathrm{~m}^{2}\right)$ & $43.5 \pm 30.9$ & $51.3 \pm 30.9$ & $44.3 \pm 25.0$ & $49.4 \pm 24.6$ & 0.4068 \\
\hline Serum sodium $(\mathrm{mEq} / \mathrm{L})$ & $141.0 \pm 2.7$ & $141.1 \pm 2.2$ & $141.9 \pm 2.2$ & $142.0 \pm 2.6$ & 0.0638 \\
\hline Serum potassium $(\mathrm{mEq} / \mathrm{L})$ & $4.21 \pm 0.65$ & $4.25 \pm 0.55$ & $4.40 \pm 0.49$ & $4.43 \pm 0.47$ & 0.0899 \\
\hline Serum chloride $(\mathrm{mEq} / \mathrm{L})$ & $105.0 \pm 4.1$ & $106.4 \pm 3.5$ & $106.1 \pm 3.2$ & $106.5 \pm 4.3$ & 0.173 \\
\hline Serum-corrected calcium (mg/L) & $9.3 \pm 0.5$ & $9.3 \pm 0.7$ & $9.4 \pm 0.6$ & $9.4 \pm 0.5$ & 0.6508 \\
\hline Serum phosphorus (mg/dL) & $3.7 \pm 1.2$ & $3.5 \pm 0.8$ & $3.6 \pm 0.6$ & $3.4 \pm 0.8$ & 0.1506 \\
\hline Serum triglyceride $(\mathrm{mg} / \mathrm{dL})$ & $146.5 \pm 75.3$ & $162.4 \pm 92.8$ & $191.0 \pm 112.3$ & $151.9 \pm 80.3$ & 0.0612 \\
\hline Serum HDL (mg/dL) & $52.8 \pm 18.5$ & $53.9 \pm 14.7$ & $51.0 \pm 17.9$ & $50.1 \pm 12.4$ & 0.6201 \\
\hline Serum LDL (mg/dL) & $126.7 \pm 46.2$ & $120.0 \pm 38.4$ & $120.1 \pm 36.4$ & $120.5 \pm 42.3$ & 0.8017 \\
\hline Serum C-reactive protein $(\mathrm{mg} / \mathrm{dL})$ & $1.1 \pm 2.6$ & $0.4 \pm 1.0$ & $0.3 \pm 0.4$ & $0.3 \pm 0.5$ & 0.0116 \\
\hline Proteinuria (g/day) & $2.5 \pm 3.2$ & $1.7 \pm 2.1$ & $1.7 \pm 2.1$ & $1.1 \pm 1.2$ & 0.0277 \\
\hline Serum IgG (mg/dL) & $1240.0 \pm 515.0$ & $1114.2 \pm 359.3$ & $1188.4 \pm 354.7$ & $1082.7 \pm 281.3$ & 0.1531 \\
\hline Serum IgA (mg/dL) & $288.1 \pm 147.0$ & $290.2 \pm 117.1$ & $301.0 \pm 98.6$ & $300.9 \pm 113.8$ & 0.9162 \\
\hline Leucocyte $\left(10^{3} / \mathrm{mL}^{3}\right)$ & $6.59 \pm 2.91$ & $6.28 \pm 1.87$ & $6.35 \pm 1.79$ & $6.88 \pm 2.05$ & 0.5003 \\
\hline Hemoglobin (g/dL) & $11.5 \pm 2.3$ & $12.1 \pm 2.5$ & $12.2 \pm 2.1$ & $12.9 \pm 2.2$ & 0.0229 \\
\hline Platelet $\left(10^{4} / \mu \mathrm{L}\right)$ & $232.9 \pm 72.6$ & $237.1 \pm 96.4$ & $217.9 \pm 70.0$ & $242.2 \pm 46.5$ & 0.3641 \\
\hline
\end{tabular}

\begin{tabular}{|c|c|c|c|c|c|}
\hline \multirow[t]{2}{*}{ (b) } & \multicolumn{5}{|c|}{ pDC quartiles (cells $/ 10^{2} \mu \mathrm{L}$ ) } \\
\hline & $\leq 256(n=52)$ & $256-417(n=53)$ & $417-560(n=52)$ & $\geq 560(n=53)$ & $p$ for trend \\
\hline Age (years) & $62.9 \pm 15.8$ & $59.2 \pm 15.6$ & $57.4 \pm 16.2$ & $51.1 \pm 15.5$ & 0.0018 \\
\hline Male, $n(\%)$ & $26,50.0 \%$ & $30,56.6 \%$ & $30,57.7 \%$ & $35,66.0 \%$ & 0.1065 \\
\hline Diabetes mellitus nephropathy, $n(\%)$ & $6,11.5 \%$ & $9,15.0 \%$ & $6,11.5 \%$ & $5,9.4 \%$ & 0.5583 \\
\hline $\operatorname{BMI}\left(\mathrm{kg} / \mathrm{m}^{2}\right)$ & $23.0 \pm 3.7$ & $23.4 \pm 3.5$ & $23.9 \pm 4.2$ & $23.7 \pm 3.7$ & 0.6773 \\
\hline Glucocorticoids user, $n(\%)$ & $5,9.6 \%$ & $8,15.1 \%$ & $4,7.7 \%$ & $7,13.2 \%$ & 0.8636 \\
\hline Serum albumin $(\mathrm{g} / \mathrm{dL})$ & $3.4 \pm 0.8$ & $3.6 \pm 0.7$ & $3.6 \pm 0.7$ & $3.6 \pm 0.8$ & 0.2038 \\
\hline Serum urea nitrogen $(\mathrm{mg} / \mathrm{dL})$ & $32.6 \pm 20.9$ & $25.6 \pm 18.4$ & $22.4 \pm 10.2$ & $19.0 \pm 15.6$ & 0.0004 \\
\hline Serum creatinine $(\mathrm{mg} / \mathrm{dL})$ & $2.3 \pm 1.7$ & $1.8 \pm 1.2$ & $1.7 \pm 1.2$ & $1.3 \pm 1.0$ & 0.0151 \\
\hline eGFR $\left(\mathrm{mL} / \mathrm{min} / 1.73 \mathrm{~m}^{2}\right)$ & $35.8 \pm 25.6$ & $45.7 \pm 26.6$ & $45.3 \pm 28.1$ & $61.4 \pm 26.1$ & $<0.0001$ \\
\hline Serum sodium $(\mathrm{mEq} / \mathrm{L})$ & $141.2 \pm 2.9$ & $141.7 \pm 2.6$ & $141.8 \pm 2.0$ & $141.2 \pm 2.3$ & 0.4612 \\
\hline Serum potassium $(\mathrm{mEq} / \mathrm{L})$ & $4.3 \pm 0.6$ & $4.3 \pm 0.6$ & $4.3 \pm 0.6$ & $4.3 \pm 0.5$ & 0.9639 \\
\hline Serum chloride $(\mathrm{mEq} / \mathrm{L})$ & $106.3 \pm 4.1$ & $106.1 \pm 4.5$ & $106.2 \pm 3.5$ & $105.3 \pm 3.0$ & 0.6347 \\
\hline Serum-corrected calcium $(\mathrm{mg} / \mathrm{L})$ & $9.3 \pm 0.6$ & $9.3 \pm 0.5$ & $9.3 \pm 0.6$ & $9.4 \pm 0.5$ & 0.9704 \\
\hline Serum phosphorus (mg/dL) & $3.7 \pm 1.3$ & $3.6 \pm 0.8$ & $3.3 \pm 0.6$ & $3.5 \pm 0.6$ & 0.1194 \\
\hline Serum triglyceride $(\mathrm{mg} / \mathrm{dL})$ & $171.3 \pm 85.6$ & $147.8 \pm 71.9$ & $184.4 \pm 113.9$ & $149.1 \pm 90.6$ & 0.1189 \\
\hline Serum HDL (mg/dL) & $48.1 \pm 14.1$ & $51.4 \pm 16.2$ & $54.0 \pm 16.0$ & $54.2 \pm 17.4$ & 0.1734 \\
\hline Serum LDL (mg/dL) & $131.4 \pm 48.0$ & $115.7 \pm 34.2$ & $119.3 \pm 43.6$ & $120.9 \pm 35.5$ & 0.2319 \\
\hline Serum C-reactive protein $(\mathrm{mg} / \mathrm{dL})$ & $1.2 \pm 2.7$ & $0.4 \pm 0.6$ & $0.2 \pm 0.4$ & $0.2 \pm 0.5$ & 0.0015 \\
\hline Proteinuria (g/day) & $2.4 \pm 3.1$ & $1.3 \pm 1.5$ & $1.6 \pm 1.9$ & $1.7 \pm 2.4$ & 0.1045 \\
\hline Serum IgG (mg/dL) & $1267.5 \pm 524.4$ & $1110.3 \pm 298.8$ & $1138.3 \pm 325.7$ & $1113.2 \pm 361.7$ & 0.125 \\
\hline Serum IgA (mg/dL) & $306.5 \pm 120.0$ & $302.2 \pm 158.0$ & $292.9 \pm 88.8$ & $278.8 \pm 101.8$ & 0.6494 \\
\hline Leucocyte $\left(10^{3} / \mathrm{mL}^{3}\right)$ & $6.88 \pm 3.01$ & $6.07 \pm 1.80$ & $6.69 \pm 1.91$ & $6.47 \pm 1.84$ & 0.2703 \\
\hline Hemoglobin (g/dL) & $11.3 \pm 2.2$ & $11.9 \pm 2.6$ & $12.2 \pm 2.2$ & $13.3 \pm 1.9$ & 0.0001 \\
\hline Platelet $\left(10^{4} / \mu \mathrm{L}\right)$ & $235.3 \pm 100.3$ & $237.1 \pm 63.3$ & $222.1 \pm 60.1$ & $235.4 \pm 64.6$ & 0.7057 \\
\hline
\end{tabular}


Table 3 (continued)

\begin{tabular}{|c|c|c|c|c|c|}
\hline \multirow[t]{2}{*}{ (c) } & \multicolumn{5}{|c|}{ tDC quartiles (cells $\left./ 10^{2} \mu \mathrm{L}\right)$} \\
\hline & $\leq 1216(n=52)$ & $1216-1439(n=53)$ & $1439-1637(n=52)$ & $\geq 1637(n=53)$ & $p$ for trend \\
\hline Age (years) & $62.5 \pm 15.1$ & $60.7 \pm 16.6$ & $55.1 \pm 14.6$ & $52.2 \pm 16.8$ & 0.0031 \\
\hline Male, $n(\%)$ & $20,38.5 \%$ & $26,49.1 \%$ & $34,65.4 \%$ & $41,77.4 \%$ & $<0.0001$ \\
\hline Diabetes mellitus nephropathy, $n(\%)$ & $8,15.4 \%$ & $7,13.2 \%$ & $7,13.5 \%$ & $4,7.6 \%$ & 0.2511 \\
\hline BMI $\left(\mathrm{kg} / \mathrm{m}^{2}\right)$ & $22.8 \pm 4.2$ & $23.5 \pm 4.0$ & $23.6 \pm 3.3$ & $23.9 \pm 3.6$ & 0.5328 \\
\hline Glucocorticoids user, $n$ (\%) & $7,13.5 \%$ & $8,15.1 \%$ & $5,9.6 \%$ & $4,7.6 \%$ & 0.2356 \\
\hline Serum albumin $(g / d L)$ & $3.3 \pm 0.7$ & $3.6 \pm 0.6$ & $3.7 \pm 0.8$ & $3.6 \pm 0.7$ & 0.0584 \\
\hline Serum urea nitrogen $(\mathrm{mg} / \mathrm{dL})$ & $31.6 \pm 19.7$ & $24.7 \pm 17.0$ & $20.1 \pm 14.0$ & $23.1 \pm 16.7$ & 0.0061 \\
\hline Serum creatinine $(\mathrm{mg} / \mathrm{dL})$ & $2.1 \pm 1.7$ & $1.8 \pm 1.4$ & $1.4 \pm 1.2$ & $1.6 \pm 1.2$ & 0.0989 \\
\hline eGFR $\left(\mathrm{mL} / \mathrm{min} / 1.73 \mathrm{~m}^{2}\right)$ & $37.7 \pm 28.6$ & $46.4 \pm 29.3$ & $54.9 \pm 25.7$ & $49.5 \pm 26.1$ & 0.0152 \\
\hline Serum sodium $(\mathrm{mEq} / \mathrm{L})$ & $141.4 \pm 3.0$ & $141.0 \pm 1.8$ & $142.3 \pm 2.0$ & $141.3 \pm 2.6$ & 0.0341 \\
\hline Serum potassium $(\mathrm{mEq} / \mathrm{L})$ & $4.3 \pm 0.6$ & $4.3 \pm 0.6$ & $4.3 \pm 0.5$ & $4.4 \pm 0.5$ & 0.4635 \\
\hline Serum chloride $(\mathrm{mEq} / \mathrm{L})$ & $105.8 \pm 3.9$ & $106.2 \pm 3.7$ & $106.9 \pm 3.7$ & $105.0 \pm 3.8$ & 0.0681 \\
\hline Serum-corrected calcium $(\mathrm{mg} / \mathrm{L})$ & $9.3 \pm 0.5$ & $9.2 \pm 0.5$ & $9.5 \pm 0.6$ & $9.3 \pm 0.6$ & 0.1358 \\
\hline Serum phosphorus (mg/dlL) & $3.8 \pm 1.2$ & $3.6 \pm 0.8$ & $3.4 \pm 0.6$ & $3.4 \pm 0.7$ & 0.063 \\
\hline Serum triglyceride (mg/dL) & $162.0 \pm 90.5$ & $147.2 \pm 69.0$ & $174.5 \pm 108.7$ & $168.5 \pm 97.2$ & 0.4692 \\
\hline Serum HDL (mg/dL) & $47.6 \pm 14.0$ & $57.5 \pm 18.3$ & $52.8 \pm 15.6$ & $49.8 \pm 14.6$ & 0.0102 \\
\hline Serum LDL (mg/dL) & $120.0 \pm 37.9$ & $121.3 \pm 36.5$ & $123.0 \pm 48.2$ & $123.0 \pm 40.8$ & 0.9777 \\
\hline Serum C-reactive protein $(\mathrm{mg} / \mathrm{dL})$ & $1.1 \pm 2.7$ & $0.4 \pm 0.7$ & $0.2 \pm 0.3$ & $0.3 \pm 0.5$ & 0.0048 \\
\hline Proteinuria (g/day) & $2.24 \pm 2.58$ & $1.36 \pm 1.95$ & $1.77 \pm 2.86$ & $1.54 \pm 1.64$ & 0.2351 \\
\hline Serum IgG (mg/dL) & $1252.5 \pm 525.5$ & $1166.7 \pm 350.6$ & $1148.0 \pm 352.8$ & $1061.9 \pm 276.3$ & 0.0956 \\
\hline Serum IgA (mg/dL) & $302.3 \pm 157.0$ & $298.0 \pm 111.1$ & $274.6 \pm 83.7$ & $305.1 \pm 116.8$ & 0.5536 \\
\hline Leucocyte $\left(10^{3} / \mathrm{mL}^{3}\right)$ & $6.59 \pm 2.85$ & $6.08 \pm 1.85$ & $6.79 \pm 1.88$ & $6.65 \pm 2.07$ & 0.3718 \\
\hline Hemoglobin $(\mathrm{g} / \mathrm{dL})$ & $11.1 \pm 2.2$ & $11.9 \pm 2.1$ & $12.9 \pm 2.1$ & $12.9 \pm 2.5$ & $<0.0001$ \\
\hline Platelet $\left(10^{4} / \mu \mathrm{L}\right)$ & $234.4 \pm 98.1$ & $225.6 \pm 67.8$ & $233.6 \pm 59.3$ & $236.4 \pm 64.7$ & 0.8839 \\
\hline
\end{tabular}

Quartile analysis showed that the level of daily proteinuria increased with decreasing blood $\mathrm{mDC}$ abs., with mean proteinuria levels (g/day) of $2.45,1.68,1.68,1.10$ for the $\mathrm{mDC}$ abs. quartiles of $\leq 445,<686,<907, \geq 907$ cells $/ 10^{2} \mu \mathrm{L}(p=0.0277)$, respectively. Compared to patients with the highest $\mathrm{mDC}$ abs., patients with the lowest $\mathrm{mDC}$ abs. were more often female. The lowest $\mathrm{mDC}$ abs. was also associated with lower serum albumin, higher C-reactive protein, a higher level of daily proteinuria and lower hemoglobin (Table 3a). Compared to patients with the highest pDC abs., patients with the lowest pDC abs. were older and had lower eGFR, higher BUN, higher serum Cr, higher C-reactive protein and lower hemoglobin (Table 3b). Compared to patients with the highest tDC abs., patients with the lowest tDC abs. were older and more often female and had lower eGFR, higher BUN, higher C-reactive protein and lower hemoglobin (Table 3c)

we revealed that the circulating $\mathrm{mDC}$ abs. was negatively associated with the level of proteinuria, independent of eGFR. These data suggest that mDCs may recruit from circulating blood to injured kidneys exhibiting heavy proteinuria, independent of the CKD stage. For accuracy, the level of proteinuria was thoroughly measured by $24-\mathrm{h}$ urine collection from patients in their admitted condition, not by spot-measuring $\mathrm{g} / \mathrm{Cr}$. This accurate measurement may contribute to the result. Second, the circulation of pDC abs. had a positive association with eGFR and no association with proteinuria. This suggests that $\mathrm{pDCs}$ are recruited into the kidney as kidney function declines, independent of the level of proteinuria. Thus, CKD stage and heavy proteinuria may regulate the distribution of each DC subset separately. Third, C-reactive protein was negatively associated with both pDC abs. and tDC abs. Fourth, male gender was positively associated with both $\mathrm{mDC}$ abs. and tDC abs.
A possible mechanism of the decrease in circulating DCs involves either DC death, a distribution shift from circulating blood to organs, or the other unknown mechanism in some kidney disease without inflammatory cell infiltration. DCs may die following infection, as in malaria [18] or severe sepsis [19], where infected DCs have been shown to undergo apoptosis. However, this mechanism seems unlikely to occur in the present CKD patients who did not have any complications of infection. Concerning the distribution shift, immature circulating mDCs are recruited to the site where the immunological response should occur, such as inflammation, tumor, hypoxic lesions, and atherosclerotic lesions, and differentiate into mature DCs [20]. For example, Yilmaz et al. [6] reported a significant reduction in circulating mDCs without a reduction in pDCs in patients with coronary artery disease. The emergence of $\mathrm{mDC}$ precursors in vulnerable plaques suggests their recruitment into atheromata as a possible reason 


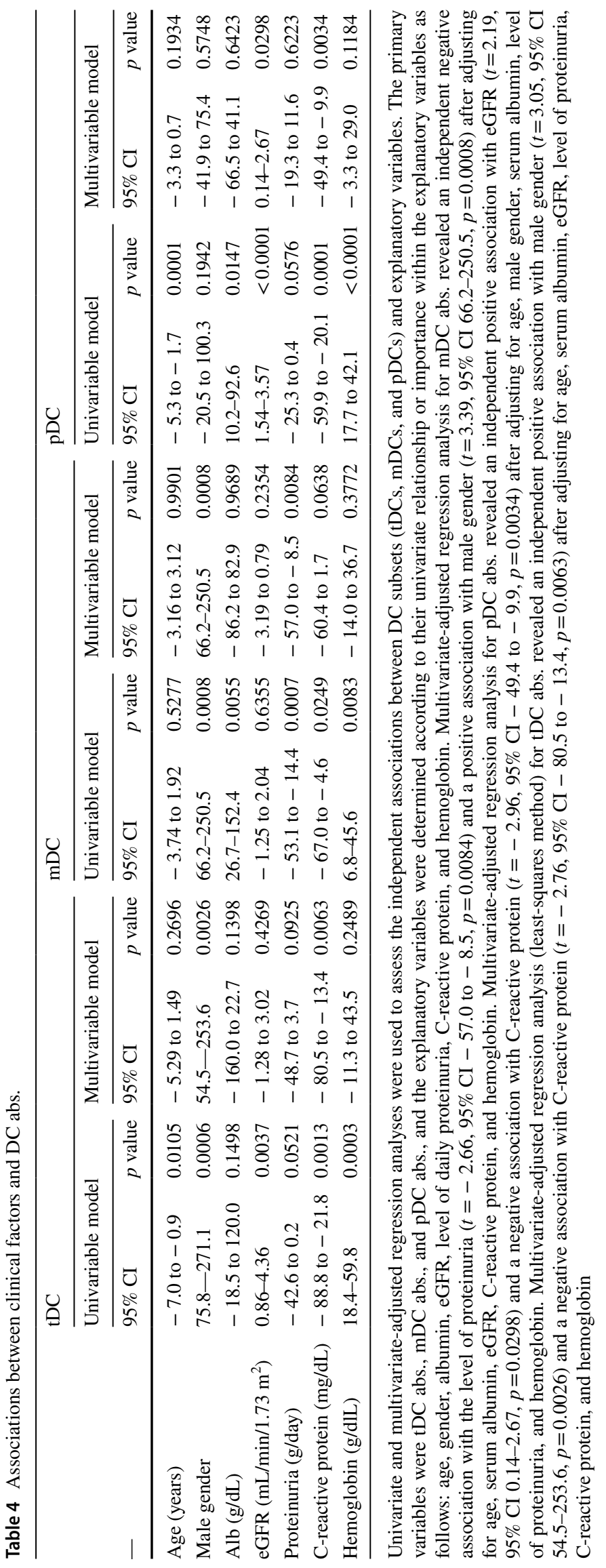


for their decrease in blood. Several reports have suggested that circulating mDCs are stimulated to migrate into the kidney glomeruli and interstitial region, which may lead to a decrease in circulating $\mathrm{mDC}$ abs [11]. In fact, both $\mathrm{mDCs}$ and pDCs were identified within the tubulointerstitium in the renal biopsies, and increased tubulointerstitial recruitment of human DCs has also been reported in lupus nephritis [3, 4], acute tubulointerstitial nephritis (ATIN) [21], renal fibrosis and CKD [22, 23]. Fiore et al. reported that $\mathrm{mDCs}$ and $\mathrm{pDC}$ that were decreased at the circulating level were recruited within the tubulointerstitial lesion in patients with active lupus nephritis. Segerer et al. [23] reported that mDC marker (DC-SIGN)-positive cells correlated significantly with serum $\mathrm{Cr}$ but not with proteinuria by simple univariate analysis in 55 renal biopsies. Their sample size might be small to detect the differences between DC-SIGN-positive cells and proteinuria, and they did not measure circulating DC subsets in the blood, so the association between circulating $\mathrm{mDCs}$ and proteinuria or DC-SIGN-positive cells in the kidney was not analyzed. The recruitment of DCs to the kidney with heavy proteinuria may be controlled by the sequential action of different chemokines as follows: the expression of monocyte chemotactic protein (MCP) by cells lining blood vessels, the production of MIP-3alpha/CCL20 by epithelial cells and the upregulation of CCR6 in response to the tissue environment [24]. Thus, chemoattractant-stimulated mDCs may migrate from circulating blood to the tubulointerstitial area in patients with heavy proteinuria. Our results suggest that this recruitment may be a common phenomenon in various kidney diseases with heavy proteinuria.

In the present study, all DC subsets showed a negative association with C-reactive protein, though a weak association was observed between C-reactive protein and mDCs (95\% CI -60.4 to $1.7, p=0.0638$ ). Similar to our study, several reports have shown a decrease in the $\mathrm{mDC}$ or/and pDC count in inflammatory diseases, such as septic shock [25], influenza A [26], and C-reactive protein-producing carcinoma [27].

The present multivariate regression analysis (210 patients) supports the proposal that renal dysfunction (decrease in eGFR) could have a negative effect on at least pDCs but not mDCs in patients with predialysis CKD, such as that in a report by Hesselink et al. [12]. Several reports have shown a reduction in the number of circulating mDCs in patients with CKD both receiving RRT and not receiving RRT [12-14]. Paul et al. [14] reported higher hsCRP concentration and the presence of diabetes mellitus had a significant negative influence on DC count but not age and eGFR. We speculate the reason were some differences of patient characteristics between ours and theirs. They selected only the patients with smaller range of eGFR $(30-60 \mathrm{ml} /$ $\left.\min / 1.73 \mathrm{~m}^{2}\right)$ than this study $\left(4.5-133.2 \mathrm{ml} / \mathrm{min} / 1.73 \mathrm{~m}^{2}\right)$, so that they could not detect a significant differences between DC number and eGFR. And this study involved only the patients who received kidney biopsy and the frequency of diabetic nephropathy was small (39.7\% in Pauls' vs $12.4 \%$ in this study) so that this study might not be able to detect a significant differences between DC number and the presence of diabetes mellitus. These discrepancy of patient characteristics might influence the results.

Human blood mDCs and pDCs express estrogen receptors (ERs) and respond to estrogens, suggesting that estrogens may contribute to the sex differences in immunity by regulating DC biology [28]. Among our 210 patients, male gender was positively associated with both $\mathrm{mDC}$ abs. and tDC abs. but was not associated with pDC abs. by multivariate regression analysis. In a healthy population, Orsini $\mathrm{G}$ et al. showed a similar male-dominant increase in $\mathrm{mDC}$ and tDC numbers, but not in pDC number, by univariate analysis in a relatively smaller sample number (44 males and 52 females), though they did not confirm their findings by a multivariate regression model [29].

Orsini et al. also observed that only the pDC abs. significantly decreased with increasing age by univariate analysis, whereas the $\mathrm{mDC}$ abs. did not show any age correlation in the subjects over 20 years [29]. We also showed that aging was associated with a decline in $\mathrm{pDC}$ number but not in $\mathrm{mDC}$ number by univariate analysis as reported by Jing et al. [30]. However, multivariate regression analysis showed no significant differences between age and each DC subset in our study.

\section{Limitation}

We conducted a cross-sectional study of 210 patients with various kidney disease and we could not detect disease specific relationship between proteinuria and circulating DCs in each renal disease.

This study included a relatively smaller number of patients with diabetic nephropathy compared to the usual CKD population given their relatively lower indication for kidney biopsy, which may influence the results. Thus, further large-scale study will be needed to clarify the relationship in each renal disease.

This study did not involve intact healthy control subjects; however, the range of eGFR was wide (4.5-133.2 ml/ $\left.\mathrm{min} / 1.73 \mathrm{~m}^{2}\right), 16$ patients with a normal eGFR $>90 \mathrm{ml} /$ $\min / 1.73 \mathrm{~m}^{2}$, and 23 patients with a normal daily proteinuria $<0.15 \mathrm{~g}$, these patient characteristics may influence the results.

We did not detect the local $\mathrm{mDC}$ by immunostaining nor by histopathological findings of tubulointerstitial inflammation and we did not measure any serum concentrations of IFN, inflammatory cytokines and chemokines, so we could not clarify the mechanism by which proteinuria influences the decrease in circulating mDCs. 
The measurement of both daily proteinuria and DC count was performed in 1 day simultaneously, although it would be preferable if more than two times simultaneous measurement of both was possible.

\section{Conclusion}

We first reported that circulating $\mathrm{mDC}$ abs. had a negative association with the level of proteinuria and no association with eGFR in CKD patients not receiving dialysis. C-reactive protein was negatively associated with both the pDC abs. and tDC abs., and male gender was positively associated with both the $\mathrm{mDC}$ abs. and $\mathrm{tDC}$ abs. in these patients.

Notably, we observed a strong reduction in mDCs, while the pDCs appears mostly unchanged in patients with heavy proteinuria. This is in line with several diseases where the involvement of DCs as a factor associated with the diseases was highlighted.

A decrease in the circulating $\mathrm{mDC}$ abs. may reflect the degree of kidney damage in CKD patients, and measurement of the blood mDC abs. may be a biomarker for renal disease activity.

\section{Compliance with ethical standards}

Conflict of interest M.I. has received scholarship funds from Baxter International, Inc. and Terumo Corporation. No other authors have any conflicts of interest to declare. No sources of funding had any direct involvement in the design or conduct of the study; the collection, management, analysis, or interpretation of data; or the preparation, review, or approval of the manuscript.

Ethical approval All procedures performed in studies involving human participants were in accordance with the ethical standards of the institutional and/or national research committee at which the studies were conducted [IRB approval number 29-032 (8648)] and with the 1964 Helsinki Declaration and its later amendments or comparable ethical standards.

Informed consent Written informed consent was not required because of the non-intervention and retrospective chart review design. We provided all individual participants a means to opt out in this study.

Open Access This article is distributed under the terms of the Creative Commons Attribution 4.0 International License (http://creativeco mmons.org/licenses/by/4.0/), which permits unrestricted use, distribution, and reproduction in any medium, provided you give appropriate credit to the original author(s) and the source, provide a link to the Creative Commons license, and indicate if changes were made.

\section{References}

1. Chowdhury F, Johnson P, Williams AP. Enumeration and phenotypic assessment of human plasmacytoid and myeloid dendritic cells in whole blood. Cytometry A. 2010;77:328-37.

2. Baumgart DC, Metzke D, Schmitz J, Scheffold A, Sturm A, Wiedenmann B, et al. Patients with active inflammatory bowel disease lack immature peripheral blood plasmacytoid and myeloid dendritic cells. Gut. 2005;54:228-36.

3. Maria NI, Davidson A. Renal macrophages and dendritic cells in SLE nephritis. Curr Rheumatol Rep. 2017;19(12):81.

4. Fiore N, Castellano G, Blasi A, Capobianco C, Loverre A, Montinaro $\mathrm{V}$, et al. Immature myeloid and plasmacytoid dendritic cells infiltrate renal tubulointerstitium in patients with lupus nephritis. Mol Immunol. 2008;45(1):259-65.

5. Cutler CW, Jotwani R, Pulendran B. Dendritic cells: immune saviors or Achilles' heel? Infect Immun. 2001;69:4703-8.

6. Yilmaz A, Weber J, Cicha I, Stumpf C, Klein M, Raithel D, et al. Decrease in circulating myeloid dendritic cell precursors in coronary artery disease. J Am Coll Cardiol. 2006;4(1):70-80.

7. Yilmaz A, Fuchs T, Dietel B, Altendorf R, Cicha I, Stumpf C, et al. Transient decrease in circulating dendritic cell precursors after acute stroke: potential recruitment into the brain. Clin Sci (Lond). 2009;19(2):147-57.

8. Ciaramella A, Salani F, Bizzoni F, Orfei MD, Caltagirone C, Spalletta G, et al. Myeloid dendritic cells are decreased in peripheral blood of Alzheimer's disease patients in association with disease progression and severity of depressive symptoms. J Neuroinflamm. 2016; 13:18.

9. Van Vre EA, Van Brussel I, Bosmans JM, Vrints CJ, Bult H. Dendritic cells in human atherosclerosis: from circulation to atherosclerotic plaques. Mediat Inflamm. 2011; 2011:941396.

10. Woltman AM, de Fijter JW, Zuidwijk K, Vlug AG, Bajema IM, van der Kooij SW, et al. Quantification of dendritic cell subsets in human renal tissue under normal and pathological conditions. Kidney Int. 2007;71(10):1001-8.

11. Hochheiser K, Engel DR, Hammerich L, Heymann F, Knolle PA, Panzer U, et al. Kidney dendritic cells become pathogenic during crescentic glomerulonephritis with proteinuria. J Am Soc Nephrol. 2011;22(2):306-16.

12. Hesselink DA, Betjes MG, Verkade MA, Athanassopoulos P, Baan CC, Weimar W. The effects of chronic kidney disease and renal replacement therapy on circulating dendritic cells. Nephrol Dial Transplant. 2005;20(9):1868-73.

13. Lim WH, Kireta S, Thomson AW, Russ GR, Coates PT. Renal transplantation reverses functional deficiencies in circulating dendritic cell subsets in chronic renal failure patients. Transplantation. 2006;81:160-8.

14. Paul K, Franke S, Nadal J, Schmid M, Yilmaz A, Kretzschmar D, et al.; GCKD Study Group. Inflammation, vitamin D and dendritic cell precursors in chronic kidney disease. Clin Exp Immunol. 2016;186(1):86-95.

15. Matsuo S, Imai E, Horio M, Yasuda Y, Tomita K, Nitta K, et al. Collaborators developing the Japanese equation for estimated GFR. Revised equations for estimated GFR from serum creatinine in Japan. Am J Kidney Dis. 2009;53:982-92.

16. Hagendorens MM, Ebo DG, Schuerwegh AJ, Huybrechs A, Van Bever HP, Bridts $\mathrm{CH}$, et al. Differences in circulating dendritic cell subtypes in cord blood and peripheral blood of healthy and allergic children. Clin Exp Allergy. 2003;33(5):633-9.

17. Upham JW, Lundahl J, Liang H, Denburg JA, O'Byrne PM, Snider DP. Simplified quantitation of myeloid dendritic cells in peripheral blood using flow cytometry. Cytometry. 2000;1(1):50-9. 
18. Pinzon-Charry A, Woodberry T, Kienzle V, McPhun V, Minigo G, Lampah DA, et al. Apoptosis and dysfunction of blood dendritic cells in patients with falciparum and vivax malaria. J Exp Med. 2013;210:1635-46.

19. Riccardi F, Della Porta MG, Rovati B, Casazza A, Radolovich $\mathrm{D}$, De Amici M, et al. Flow cytometric analysis of peripheral blood dendritic cells in patients with severe sepsis. Cytom B Clin Cytom. 2011;80:14-21.

20. Weisheit CK, Engel DR, Kurts C. Dendritic cells and macrophages: sentinels in the kidney. Clin J Am Soc Nephrol. 2015;7(10):1841-51.

21. Cheng M, Gu X, Herrera GA. Dendritic cells in renal biopsies of patients with acute tubulointerstitial nephritis. Hum Pathol. 2016;54:113-20.

22. Kassianos AJ, Wang X, Sampangi S, Muczynski K, Healy H, Wilkinson R. Increased tubulointerstitial recruitment of human CD141 (hi) CLEC9A (+) and CD1c (+) myeloid dendritic cell subsets in renal fibrosis and chronic kidney disease. Am J Physiol Renal Physiol. 2013;15(10):F1391-401.

23. Segerer S, Heller F, Lindenmeyer MT, Schmid H, Cohen CD, Draganovici D, et al. Compartment specific expression of dendritic cell markers in human glomerulonephritis. Kidney Int. 2008;74(1):37-46.

24. Vanbervliet B, Homey B, Durand I, Massacrier C, Aitt-Yahia S, de Bouteiller O, et al. Sequential involvement of CCR2 and CCR6 ligands for immature dendritic cell recruitment: possible role at inflamed epithelial surfaces. Eur J Immunol. 2002;32(1):231-42.
25. Grimaldi D, Louis S, Pène F, Sirgo G, Rousseau C, Claessens YE, et al. Profound and persistent decrease of circulating dendritic cells is associated with ICU-acquired infection in patients with septic shock. Intensive Care Med. 2011;37(9):1438-46.

26. Lichtner M, Mastroianni CM, Rossi R, Russo G, Belvisi V, Marocco R,et al. Severe and persistent depletion of circulating plasmacytoid dendritic cells in patients with 2009 pandemic H1N1 infection. PloS One. 2011;6(5):e19872.

27. Frenzel H, Pries R, Brocks CP, Jabs WJ, Wittkopf N, Wollenberg B. Decreased migration of myeloid dendritic cells through increased levels of C-reactive protein. Anticancer Res. 2007;27(6B):4111-5.

28. Escribese MM, Kraus T, Rhee E, Fernandez-Sesma A, Lopez CB, Moran TM. Estrogen inhibits dendritic cell maturation to RNA viruses. Blood. 2008;112:4574-84.

29. Orsini G, Legitimo A, Failli A, Massei F, Biver P, Consolini R. Enumeration of human peripheral blood dendritic cells throughout the life. Int Immunol. 2012;24(6):347-56.

30. Jing Y, Shaheen E, Drake RR, Chen N, Gravenstein S, Deng Y. Aging is associated with a numerical and functional decline in plasmacytoid dendritic cells, whereas myeloid dendritic cells are relatively unaltered in human peripheral blood. Hum Immunol. 2009;70(10):777-84.

Publisher's Note Springer Nature remains neutral with regard to jurisdictional claims in published maps and institutional affiliations. 